\title{
The Effect of Increased Cognitive Demand on Auditory Processing Assessment
}

DOI: $10.3766 /$ jaaa.16152

\author{
Kathryn Hamlyn* \\ Katie Welldon* \\ Benjamin Clark* \\ Willem van Steenbrugge* \\ Sarosh Kapadia*
}

\begin{abstract}
Background: Auditory processing (AP) is commonly regarded as the perceptual processing of auditory information in the central nervous system. However, the degree to which higher level cognitive processes are involved in AP or its disorders is contentious. Furthermore, there is little evidence regarding the effects of nonauditory cognitive processes on the various tests of AP in common clinical usage and thus on clinical diagnoses of auditory processing disorder.
\end{abstract}

Purpose: To determine the effects of increased cognitive demand, generated by using a dual-task paradigm, on performance on different AP tests and types of AP tests in common clinical usage. In addition, to investigate the relationship between executive function and changes in AP test performance associated with increased cognitive demand.

Research Design: Counterbalanced repeated measures design, with assessment of AP test performance both on its own and in a dual-task paradigm designed to increase cognitive demand.

Study Sample: Twenty-nine young adults, with no reported hearing, learning, language or attention difficulties, English as first language, and hearing and middle-ear status within normal limits.

Data Collection and Analysis: Testing was completed within a single 90-min session. A selection of standard AP tests, representing both adaptive and nonadaptive tests, as well as tests employing difference scores, was administered. These were Competing Sentences Test, Dichotic Digits Test, Frequency Pattern Test (nonadaptive tests); and Listening in Spatialized Noise-Sentences test, conditions "samevoice, $0^{\circ}$ ", "different-voice, $0^{\circ}$ ", and "same-voice, 90" (adaptive tests), from which the difference scores "talker advantage" and "spatial advantage" were also derived. Each AP test was completed on its own (alone condition), and simultaneously with a visually presented task (dual-task condition). Executive function was assessed using the phonemic subtest of the Verbal Fluency Test. Nonparametric statistical test procedures were used.

Results: All five AP measures obtained from the nonadaptive tests showed a significant performance decrement in the dual-task condition compared with the alone condition, with one exception because of a strong ceiling effect. By contrast, none of the three AP measures obtained from the adaptive tests showed a significant performance decrement in the dual-task condition. Furthermore, neither of the two AP measures based on difference scores showed a significant performance decrement, but this finding simply reflects the lack of significant decrements in the relevant raw scores. Consistent with past reports of associations between executive function and AP performance, a significant positive correlation was found between executive function scores and performance on the Dichotic Digits Test. However, there were no significant correlations between executive function scores and changes in AP test scores between alone and dual-task conditions. edu.au

*Department of Speech Pathology and Audiology, Flinders University, Adelaide, Australia

Corresponding author: Sarosh Kapadia, Speech Pathology and Audiology, Flinders University, Adelaide 5001, Australia; Email: sarosh.kapadia@flinders. 2014.

Portions of this study were presented at AudiologyNOW!, Orlando FL, March 2014, and the World Congress of Audiology, Brisbane, Australia, May 
Conclusions: Performance on commonly used nonadaptive tests of AP was significantly compromised by the increased cognitive demand resulting from the dual-task paradigm. By contrast, performance on AP measures obtained by adaptive test procedures was not significantly affected. Further investigation of the resilience to increased cognitive demand of the adaptive tests used here, and other adaptive tests of AP, is warranted. Results from this study support the further development of computerized adaptive tests of AP for use in clinical test batteries.

Key Words: auditory processing, auditory processing disorder, cognitive demand, dual task, executive function

Abbreviations: AP = auditory processing; APD = auditory processing disorder; CST = Competing Sentences Test; DDT = Dichotic Digits Test; DV = different voice; FPT = Frequency Pattern Test; LiSN-S = listening in spatialized noise-sentences; NVIQ = nonverbal intelligence quotient; SRT = speech reception threshold; SV $=$ same voice; VFT $=$ Verbal Fluency Test

\section{INTRODUCTION}

$\mathrm{A}$ uditory processing (AP) is commonly defined as the perceptual processing of auditory information in the central nervous system (ASHA, 2005a; AAA, 2010). Individuals who have difficulty processing auditory information effectively, despite having normal hearing sensitivity and intellectual capacity, may be diagnosed with an auditory processing disorder (APD) (Chermak et al, 2002; Cameron and Dillon, 2007; Dawes and Bishop, 2009). However, the degree to which higher level cognitive factors may influence AP and APD is a topic of vigorous ongoing debate (e.g., Jerger and Musiek, 2000; Gates et al, 2010; Moore et al, 2010; Tomlin et al, 2015), with important theoretical and clinical implications. A substantial part of this debate hinges on the definitions of AP and APD, and despite a number of published position statements and perspectives (e.g., Jerger and Musiek, 2000; ASHA, 2005a; BSA, 2011; Cacace and McFarland, 2013), there is currently no real consensus on these definitions (e.g., CISG, 2012; Moore et al, 2013; Vermiglio, 2014; de Wit et al, 2016).

A particular point of contention is the role of higherorder (nonauditory) factors in APD. For example, ASHA (2005b, p. 2) states that APD "requires demonstration of a deficit in the neural processing of auditory stimuli that is not due to higher order language, cognitive, or related factors." However, based on a large study of AP in children, Moore et al (2010, p. e382) assert that "APD is primarily an attention problem." Similarly, Gates et al (2010) argue that central auditory dysfunction (in older adults) is not distinct from cognitive dysfunction.

A related, though not identical, issue concerns the clinical assessment and therefore diagnosis of APD. In practice, an individual suspected of having an APD is assessed using a battery of tests that examine multiple areas of AP (e.g., Musiek and Chermak, 2007; Sharma et al, 2009). These tests are almost universally behavioral in nature (Jerger et al, 2002). As behavioral tests are potentially subject to higher-order confounds (e.g., motivational, attentional, or other cognitive factors), it is important for the assessing clinician to identify and take account of any such confounds (ASHA, 2005a). The present study therefore seeks to investigate the extent to which higher cognitive processes affect different tests of AP in common clinical usage and thus clinical diagnoses of APD. It is argued that such an understanding would be of value, regardless of a precise definition of APD and regardless of whether performance on tests of AP accurately reflects that definition.

\section{Tests of AP}

There is currently no gold-standard diagnostic test battery of APD; however, as APD is not isolated to an impairment of a single process within the auditory system, it is recognized that a variety of tests need to be used to ensure that multiple areas of AP are assessed (Musiek and Chermak, 2007; Sharma et al, 2009). From the particular perspective of the present study, two important distinctions are made: (a) between tests that are adaptive or nonadaptive in nature and (b) between tests that do or do not employ control conditions.

In a nonadaptive test, the level of difficulty of each trial is predetermined (Leek, 2001). In practice, the vast majority of clinical tests of AP are nonadaptive and assess performance at a single level of difficulty only. Thus, the ability of such tests to optimally engage a participant and to avoid effects of fatigue, boredom or discouragement depends on the difficulty level chosen by the developers of the test and on the homogeneity of test ability of the target population (O'Beirne et al, 2012). In an adaptive test, the level of difficulty of each trial is dependent on the participant's response to one or more previous trials (Leek, 2001). This permits most of the testing to be conducted at a specified level of performance (e.g., $50 \%$ correct), regardless of differences in test ability across participants. Adaptive measures in speech intelligibility tests are used for the ease in which the speech reception threshold (SRT) can be calculated, and the ability to minimize the within-participant SRT standard deviation (Cameron and Dillon, 2007). Major advantages of adaptive testing also include the potential reduction of floor 
and ceiling effects, and a reduced susceptibility to confounding factors such as motivation, attention, and fatigue (Jerger and Musiek, 2000; O'Beirne et al, 2012).

A second important distinction between AP tests is between tests that do or do not employ control conditions. Tests that employ control conditions typically express results in terms of a difference score (i.e., results are calculated by determining the difference in test scores across a pair of test conditions, which differ only in the critical parameter of interest). The influences of higher-order language, learning, and communication factors on test results are expected to be reduced in tests that employ control conditions as these factors are common to each test condition (Cameron et al, 2011). Once again, the use of control conditions is rare in clinical tests of AP. Development of AP tests that employ both adaptive techniques and control conditions could reduce the potential for confounds unrelated to AP to influence its assessment.

\section{Cognitive Factors and AP}

Several studies have examined the relationship between AP and cognitive factors. Table 1 summarizes a number of studies that have used broadly comparable approaches, noting the specific AP tests used and the broad findings of particular relevance to the present study. Table 1 and the following discussion also note the relevant psychometric properties of the AP tests used, highlighting any differences in findings between AP tests that were adaptive or nonadaptive, and between tests that did or did not employ control conditions.

McArthur and Bishop (2004) investigated frequency discrimination ability using a computerized adaptive procedure (with no control condition), in individuals with specific language impairment and a control group. They found that individuals with poor frequency discrimination also had significantly poorer memory for temporal order than those with normal frequency discrimination. (Memory for temporal order was regarded as a "task-related ability" for the frequency discrimination task used in this study.) However, there were no significant differences between the groups with respect to the attention measures or nonverbal intelligence quotient (NVIQ).

In studying the comorbidity of APD, reading disorder and language impairment in school-aged children with suspected or diagnosed APD, Sharma et al (2009) employed a range of nonadaptive clinical AP measures, one of which also employed a control condition. They reported significant correlations between auditory attention and/or auditory memory and two of the AP measures, but not with the other two (which included the measure that used a control condition).

Perhaps the most detailed and extensive of the studies in this area is that of Moore et al (2010), who assessed 1,469 randomly selected children aged 6-11 yrs on a battery of AP and cognitive tests. They used purpose-developed, rather than clinically used, tests of AP, which were all adaptive, and included tests that did and did not employ control conditions. The cognitive measures of most relevance to our study were a measure of "intrinsic" attention based on performance variability on the AP tests, separate measures of "extrinsic" (sustained) auditory and visual attention, digit and phonological memory, and NVIQ. The key finding of this study was that poorer AP test performance was closely associated with greater variability in performance (poorer intrinsic attention). However, perhaps surprisingly, this association also applied to the AP measures derived from control conditions (which are expected to be resistant to attentional factors). Moore et al (2010) also found that auditory sustained attention was not related to any of their AP measures, but visual sustained attention was significantly related to some of their raw AP measures. Finally, these authors found that NVIQ, and digit and phonological memory were significantly correlated with raw AP scores, but, as expected, were generally not correlated with the AP scores derived from control conditions. The major conclusion of Moore et al (2010) was that poor performance on AP tasks may be due to problems with attention rather than with auditory (sensory) processing per se.

Wilson et al (2011) reported data on the relationship between four commonly used clinical AP test measures (all nonadaptive) and a suite of auditory memory measures, in children aged 6-11 who had been referred for AP assessment. In general, auditory memory measures were significantly correlated with the AP measures with little to no linguistic load.

Ahmmed et al (2014) assessed children aged 6-11 referred for APD assessment due to listening difficulties, using a common clinical test battery (nonadaptive measures), as well as the raw AP measures used by Moore et al (2010) (adaptive measures). This study was primarily a factor analysis of the various outcomes measured. However, from the perspective of the present study, it found, broadly, that auditory memory measures were significantly correlated with most AP measures, attention measures were significantly correlated with a few, and NVIQ was not significantly correlated with any AP measure. There was no major distinction in this regard evident between the adaptive and the nonadaptive measures.

In a study focusing primarily on AP and attention, Gyldenkærne et al (2014) assessed AP, sustained attention and NVIQ in children aged 7-12 with reported listening difficulties. Standard clinical tests of AP were used, which were similar to those of Sharma et al (2009) (all nonadaptive and one with a control condition). Sustained attention and NVIQ were found to be significantly correlated with two of the AP measures 


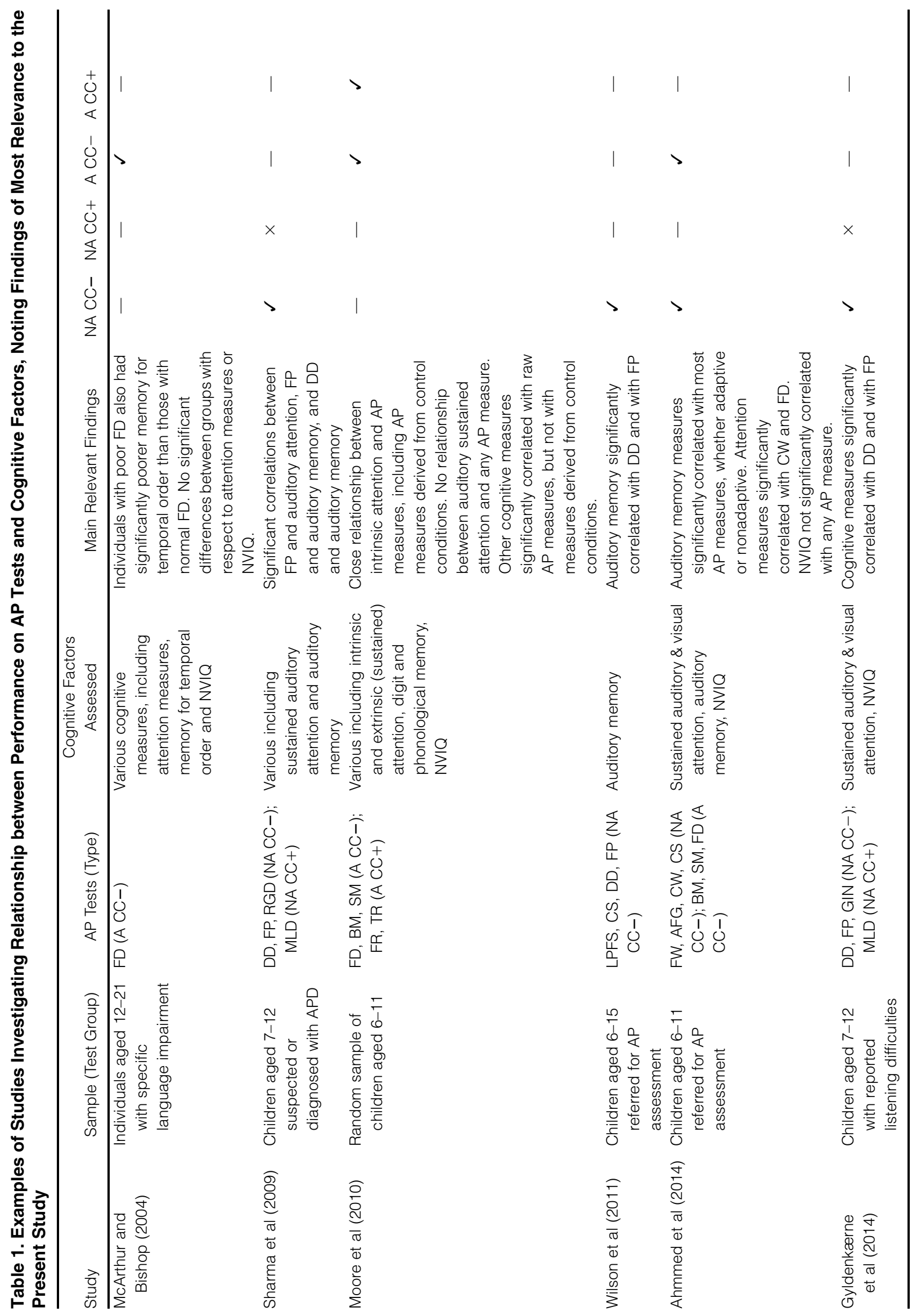




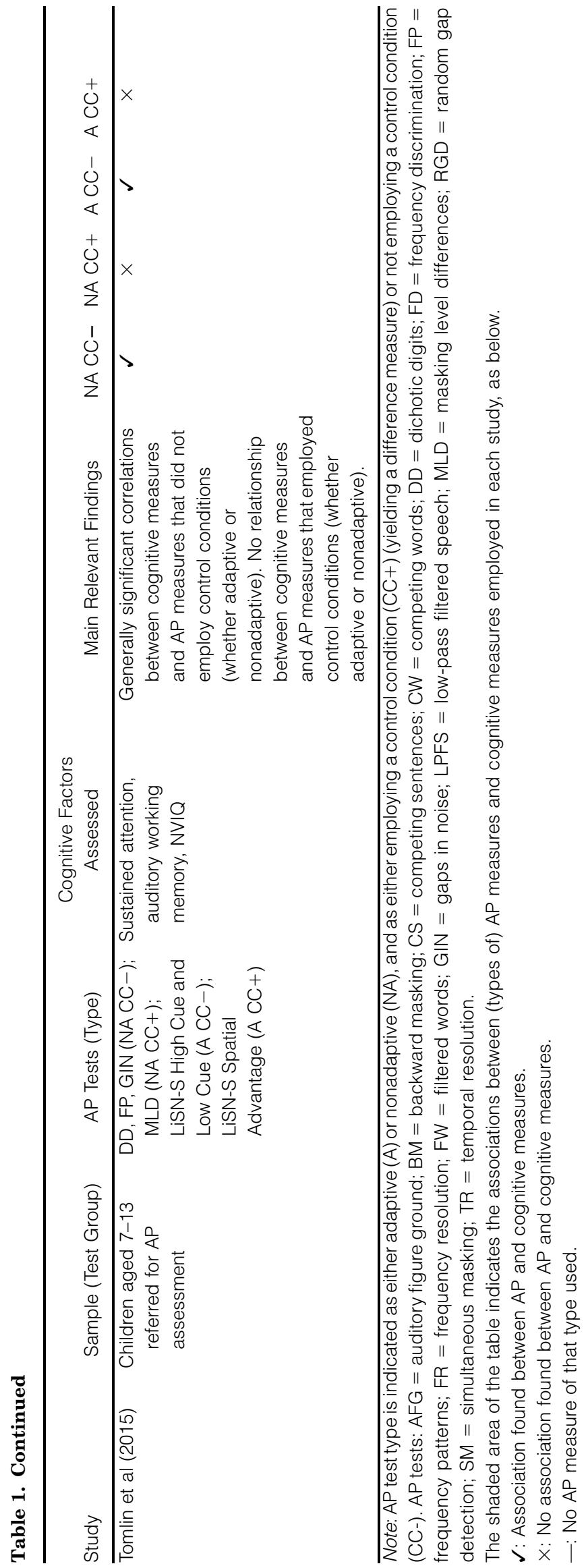

that lacked a control condition, but not with the other two (one of which employed a control condition).

Finally, Tomlin et al (2015) reported on 105 children aged 7-13 yrs referred to an audiology clinic for a clinical APD assessment, and an age-matched control group. These authors assessed AP abilities using a range of clinical tests that included adaptive and nonadaptive tests (and two measures derived from control conditions); and cognitive abilities of sustained attention, auditory working memory and NVIQ. In general, they found cognitive measures were significantly correlated with AP measures that did not employ control conditions (whether adaptive or nonadaptive), but not with AP measures that employed control conditions (whether adaptive or nonadaptive).

In considering the studies discussed previously and in Table 1 together, some possible trends with respect to the specific AP tests employed may be discerned, for example, the dichotic digits and frequency pattern tests appear to be more frequently related to the cognitive factors studied than do the gap detection tests. Furthermore, from the perspective of the psychometric properties of the tests used, the studies on the whole suggest that results from tests that employ control conditions are indeed less related to the cognitive factors studied than are tests that do not employ control conditions. However, no such distinction is evident between adaptive versus nonadaptive tests. The general conclusion of most authors of the above studies is that performance on AP tasks may be associated with cognitive factors, with some differences of opinion on the relative importance of the association, and on the causal nature of the cognitive factors. Given the wider debate in the field over the influence of cognitive factors on AP and APD, there remains a need to identify which AP tests are more resilient to the effects of cognitive factors and why, as previously argued by Dawes and Bishop (2009).

Furthermore, in studying the relationship between AP test performance and cognitive factors, AP and cognitive abilities are typically assessed separately, and it is then determined if an association between scores on each of these measures exists. However, the success of this method relies on the choice of a measure of cognitive ability being applicable to AP task performance (at a different point in time), and the approach may not provide information regarding the direct effect of higher-order cognitive function on AP task performance.

\section{The Dual-Task Paradigm}

An alternative approach to assessing the possible influence of higher-order confounds on task performance is the use of a dual-task paradigm (Pashler, 1994). The dual-task paradigm requires the completion of two 
different tasks simultaneously—the primary task and a competing secondary task (Troyer and Craik, 2000). Typically, unless participants are instructed to prioritize one task at the expense of the other, performance in both tasks is poorer than when completed singly. An analysis of such performance changes may provide insight into various aspects of each task (e.g., Troyer and Craik, 2000; Boot et al, 2005; McCulloch, 2007).

For instance, Heinrich et al (2008) used the dual-task paradigm to demonstrate the effect of increased cognitive demands on short-term auditory memory. The study found that short-term auditory memory, as assessed by the ability to correctly recall word pairs, was reduced when performed simultaneously with a visually presented secondary task, compared with when the auditory task was performed alone (Heinrich et al, 2008).

Models of dual-task interference generally invoke the sharing of (finite) resources such as attention, processing capacity, and working memory (Pashler, 1994). Use of the dual-task paradigm to increase the cognitive demands placed on participants thus affords a controllable, experimental approach to examine the relationship between AP and such cognitive factors. The degree of reduction in AP test performance under dual-task conditions would indicate susceptibility to such factors, and allow a systematic comparison of this susceptibility across AP tests and types of AP tests. Although dual tasks have been used within auditory research to assess "listening effort" (reviewed by McGarrigle et al, 2014), there do not appear to be any reports to date that describe the direct impact of a competing task on AP test performance.

\section{Executive Function}

Executive function is an umbrella term referring to the control of multiple cognitive processes that allow us to perform a range of tasks, including planning and organizing daily activities, problem solving and decision making, and modulating selective attention when confronted with novel situations. Executive function comprises at least three components (see Diamond, 2013): (a) inhibition of irrelevant, interfering stimuli or responses; (b) working memory, that is, the ability to temporarily store and maintain relevant information for comparison or decision making; and (c) cognitive flexibility, that is, the ability to switch smoothly between one stimulus or response to the next.

The term executive function is often used to encapsulate these three components, all of which are important in the modulation and control of a wide range of cognitive functions. In other words, the term executive function also refers to a general modulating device enabling the cognitive processes that are responsible for the control and regulation of multiple lower level functions.
Executive function is thought to facilitate the effective use of basic sensory, motor, perceptual and linguistic skills, and allow self-regulated, future-oriented and goal-directed behavior (Cheung et al, 2004). Both the allocation and control of attention is thought to be mediated by executive function (McCulloch, 2007; Diamond, 2013).

Foster et al (2013) reported a relationship between auditory temporal processing and inhibition, planning and auditory memory. The study used electrophysiological recordings of the event-related potential mismatch negativity response to an auditory stimulus as a measure of temporal processing, and compared these results with measures of cognition and executive function (Foster et al, 2013). The findings indicated significant positive correlations between auditory temporal processing, and measures of auditory (verbal) memory and executive function. Gates et al (2010) also found a significant relationship between measures of executive function and AP, with composite executive function scores explaining a reported $8-21 \%$ of variance in AP test scores. These studies suggest that executive function may be directly related to AP test performance. However, the role of executive function in allocation and control of attention and cognitive flexibility also suggests that it may be particularly related to the impact of a competing task on AP test performance, in a dualtask paradigm. This relationship has not previously been studied.

\section{Aims}

The aims of this study were:

- To determine the effect of increased cognitive demand on performance on a variety of AP tests in common clinical usage by using a dual-task paradigm

- To determine whether the effects of increased cognitive demand are different for AP tests with different psychometric properties; that is, adaptive versus nonadaptive AP tests, and tests that employ control conditions versus tests that do not

- To investigate whether measures of executive function predict the degree to which AP test performance is affected by the increased cognitive demand in the dual-task condition

\section{METHODS}

\section{Participants}

Participants were 29 adults aged between 18 and 40 yrs, with no reported hearing difficulties and English as their first language. Exclusion criteria were hearing thresholds $>20 \mathrm{~dB}$ HL at any octave frequency between 250 and $8000 \mathrm{~Hz}$, abnormal middle ear function as 
indicated by Type B or C tympanograms, otological abnormalities, or previous diagnosis of learning, language, or attention deficits. The mean age of the participants was $29.7 \mathrm{yrs} ; 20$ were female and 9 were male. Mean three-frequency pure-tone averages (i.e., average of thresholds at 500, 1000, and $2000 \mathrm{~Hz}$ ) were $2.8 \mathrm{~dB}$ HL in the right ear, and $2.6 \mathrm{~dB} \mathrm{HL}$ in the left.

\section{Audiological Procedures}

All testing was completed within a single session of approximately $90 \mathrm{~min}$, commencing with a brief questionnaire covering hearing, listening, speech/language, learning, and attention difficulties. Otoscopic examination was performed to determine any otological abnormalities. Pure-tone air-conduction thresholds were obtained between 250 and $8000 \mathrm{~Hz}$ bilaterally, using an Aurical Plus (version 3.09) computer-controlled audiometer with Telephonics TDH 39P headphones in a sound-attenuated booth. Tympanometry was performed using a Grason-Stadler GSI TympStar.

\section{Auditory Processing Tests}

AP ability was measured using both adaptive and nonadaptive tests in common use and frequently reported in the literature. All AP tests were administered according to their standard clinical protocol in a sound-attenuated booth, unless otherwise stated.

Three nonadaptive AP tests, which represent relatively heavy, light and no linguistic load, were used. These were, respectively, the Willeford Competing Sentences Test (CST), Musiek Dichotic Digits Test (DDT) version 2 (double), and Musiek Frequency Pattern Test (FPT). These tests were administered via the same computer-controlled audiometer and headphones mentioned previously. Each CST response was scored as correct only if the entire sentence was repeated correctly. The right ear was always tested first, followed by the left ear. The target sentence was presented at $35 \mathrm{~dB}$ SL with reference to the three-frequency puretone average for the test ear (minimum presentation level of $40 \mathrm{~dB} \mathrm{HL}$ ), and the competing sentence presented at $15 \mathrm{~dB}$ above the target. For the DDT, twenty sets of two-digit pairs were presented at $50 \mathrm{~dB} \mathrm{HL}$, with scoring allowing for a right ear and left ear result to be obtained. Participants were not required to repeat the digits in a particular order. The FPT was presented at $50 \mathrm{~dB}$ SL binaurally under headphones, with reference to the $1000 \mathrm{~Hz}$ pure-tone threshold averaged between ears. Twenty-five sets of tone triplets were presented, with the high tone at $1122 \mathrm{~Hz}$ and the low tone at 880 $\mathrm{Hz}$. Responses were provided verbally and scored correct only if the pattern was reported correctly (e.g., "high, high, low") in the correct order. Reversed responses were scored as errors. All nonadaptive AP tests results were recorded as percentage correct scores.

The adaptive measures of AP involved three adaptive subtests of the Listening in Spatialized Noise-Sentences (LiSN-S) test (PHONAK, 2009) administered under Sennheiser HD215 headphones. The LiSN-S test assesses recognition of target sentences in the presence of two-talker competing speech, which may either be in the same voice (SV) or a different voice (DV) as the target sentences. Furthermore, target and competing speech may either be spatially colocated $\left(0^{\circ}\right)$ or separated $\left(90^{\circ}\right)$. (The LiSN-S is conducted under headphones, spatial separation is simulated by the test software using head-related transfer functions [Cameron and Dillon, 2007].) In the present study, the LiSN-S adaptive subtests for conditions SV0, DV0, and SV90 were administered. Results for each subtest were recorded as SRTs in dB SNR (signal to noise ratio) for 50\% correct performance, as determined by the adaptive procedure. These SRTs were further used to derive the difference measures of "talker advantage" and "spatial advantage," using the $\mathrm{SV}^{\circ}$ condition as the control condition. Talker advantage was calculated as the difference in $\mathrm{dB}$ between the SV0 and $\mathrm{DV} 0^{\circ}$ SRTs, and spatial advantage as the difference in $\mathrm{dB}$ between the $\mathrm{SV0}$ and $\mathrm{SV} 90^{\circ} \mathrm{SRT}$.

\section{Dual-Task Condition}

Each AP test was completed alone, as well as in the dual-task condition. The secondary task used in the dual-task condition was the "3-odds" task described by Heinrich et al (2008), and implemented using the DMDX software (Forster and Forster, 2003). Participants were presented a continuous stream of single digits on a laptop computer screen at a rate of approximately $1 /$ sec, and instructed to press a key every time three consecutive odd digits appeared. A brief error message was displayed immediately for either incorrect or missed responses. Errors, in the form of both incorrect key presses and misses, were recorded by the software. In the dualtask condition, participants were instructed to complete both the auditory and visual task to the best of their ability and encouraged and reminded to do so during testing. (In contrast, when dual tasks are employed to assess listening effort, participants are typically instructed to prioritise performance of the primary task, such that performance on this task is maintained, relative to the alone condition.) The "3-odds" task was manually terminated when the coadministered AP test ran to completion. This took between 1.8 and $3.1 \mathrm{~min}$ on average, depending on the AP test involved. The "3-odds" task was also completed as a standalone test, to obtain baseline scores for the task. Participants completed this test for approximately $2.4 \mathrm{~min}$ (144 digit presentations). To control for the variable lengths of the runs of the " 3 -odds" task, the number of errors committed in each run was 
normalized by dividing by the total number of digits presented in that run.

\section{Executive Function Assessment}

Executive function was assessed using the phonemic subtest of the Verbal Fluency Test (VFT), which assesses the ability to spontaneously generate words under restricted search conditions (Strauss et al, 2006). Participants were instructed to list as many words as they could in $60 \mathrm{sec}$ beginning with a certain letter, with the letters F, A, and S used, in that order, for all participants. Participants responded verbally, and their responses were recorded using a digital voice recorder.

Phonemic fluency is considered to be a measure of executive function because generating words on the basis of an orthographic criterion (a letter) is a novel, unusual task (unlike generating animal names, for instance) that also involves (a) keeping the instruction of the task in mind as well as (b) inhibiting words previously generated during the task, which (c) therefore have to be in working memory, as well as being able to (d) suppress irrelevant responses (Henry and Crawford, 2004). In short, the efficient completion of the phonemic VFT is thought to rely on multiple executive function skills, including initiating, planning, clustering, switching, and working memory (Strauss et al, 2006).

\section{Control of Order and of Learning Effects}

To control for potential participant fatigue, and/or learning effects, the order in which participants completed the tests was counterbalanced. Complete counterbalancing across all tests was not possible for the number of tests and participants. Therefore, all adaptive tests were grouped together, as were all nonadaptive tests (which also included the tests with control measures). A partially counterbalanced design was used, with eight sequences of test group/condition order, shown in Table 2. This was designed to avoid the potential of order effects (a) confounding the comparison of alone versus dual-task results for each test, and (b) confounding the comparison of results between the groups of tests. As shown in Table 2, this design ensured that approximately half of the participants included (15 of 29) completed the nonadaptive AP tests in the alone condition before the dual-task condition. Similarly, approximately half of the participants (15 of 29) completed the adaptive AP tests in the alone condition before the dual-task condition. Finally, approximately equal numbers of participants completed the adaptive tests in the dual-task condition before the nonadaptive tests in the dual-task condition $(n=15)$, as completed the nonadaptive tests in the dual-task condition before the adaptive tests in the dual-task condition $(n=14)$. The order of tests within each group (adaptive and nonadaptive) was not counterbalanced.

To minimize learning effects on the CST, the target sentence list was changed for participants' second completion of the test (i.e., list A to B or vice versa). This was also counterbalanced among participants, to minimize any list effects, with approximately equal numbers of the participants who completed the CST in the alone condition first (15 of 29) being presented with list $\mathrm{A}$ as the target $(n=7)$ as list $B(n=8)$. Similarly, approximately equal numbers of the participants who completed the CST in the dual-task condition first (14 of 29) were presented with list A as the target $(n=7)$, as list $B(n=7)$. The test lists for the DDT and FPT were the same for both repeats, as minimal recall of the actual test material on repeat testing was anticipated for these tests.

\section{Statistical Analysis}

Statistical analysis was conducted using IBM SPSS Statistics version 21. Normality tests indicated that

Table 2. Counterbalancing of Order in Which Adaptive (A) and Nonadaptive (NA) Groups, and Alone and Dual Conditions of Tests Were Conducted across the 29 Participants

\begin{tabular}{|c|c|c|c|c|}
\hline Test Group/Condition Order & $\mathrm{N}$ & $\begin{array}{l}\text { N (NA Alone } \\
\text { Before Dual) }\end{array}$ & $\begin{array}{c}\text { N (A Alone } \\
\text { Before Dual) }\end{array}$ & $\begin{array}{c}\mathrm{N} \text { (A in Dual } \\
\text { Before NA in Dual) }\end{array}$ \\
\hline NA alone - $\mathrm{A}$ alone - NA dual - $\mathrm{A}$ dual & 4 & (4) & (4) & (0) \\
\hline A alone - NA alone - A dual - NA dual & 4 & (4) & (4) & (4) \\
\hline A dual - NA dual - A alone - NA alone & 4 & (0) & (0) & (4) \\
\hline NA dual - A alone - NA alone - A dual & 4 & (0) & (4) & (0) \\
\hline A dual - NA alone - A alone - NA dual & 4 & (4) & (0) & (4) \\
\hline NA dual - A dual - NA alone - A alone & 3 & (0) & (0) & (0) \\
\hline A alone - NA dual - A dual - NA alone & 3 & (0) & (3) & (0) \\
\hline NA alone - A dual - NA dual - A alone & 3 & (3) & (0) & (3) \\
\hline Totals & 29 & 15 & 15 & 15 \\
\hline
\end{tabular}

Note: As shown, half the participants completed the nonadaptive AP tests in the alone condition before the dual-task condition; half the participants completed the adaptive AP tests in the alone condition before the dual-task condition; and half the participants completed the adaptive tests in the dual-task condition before the nonadaptive tests in the dual-task condition. 
scores for many of the nonadaptive AP tests were not normally distributed (being generally clustered close to the maximum score). Errors committed in the secondary ("3-odds") task were also generally not normally distributed. Therefore, nonparametric tests were used throughout, namely the Wilcoxon signed-rank test for paired comparisons, and Spearman's rho for correlations. All significance testing ( $p$ values) are for twotailed testing.

The study was approved by the Southern Adelaide Clinical Human Research Ethics Committee.

\section{RESULTS}

\section{AP Test Performance in Alone and Dual- Task Conditions}

Figure 1 shows the mean scores (percentage correct) for the nonadaptive tests of AP in alone and dual-task conditions. Mean scores in the alone condition are within the normal range for an adult population for all these tests, that is, at least $90 \%$ in each ear for the CST and the DDT, and at least 78\% for the FPT (Musiek, 1983; Musiek, 1994; Bellis, 2003). Wilcoxon signed-rank tests showed a significant decrease in scores in the dual-task condition compared with the alone condition in all of the nonadaptive tests except CST right ear. Significant differences were found for CST left ear $(Z=2.57, p<0.05)$, DDT right ear $(Z=2.17, p<0.05)$, DDT Left ear $(Z=$ $3.50, p<0.001)$ and FPT $(Z=3.26, p<0.005)$, but no significant difference was found for CST right ear $(Z=$ $0.33, p>0.05$ ). (The mean score in the dual-task condition is below the normal [alone condition] range for an adult population in only one test, CST Left ear. However, the main focus of this study was the relative sensitivity to increased cognitive demand of different AP tests, rather than whether scores in the dual-task condition fell outside the normal range.)

Figure 2 shows the mean scores for the adaptive tests of AP in the alone and dual-task conditions. Scores are

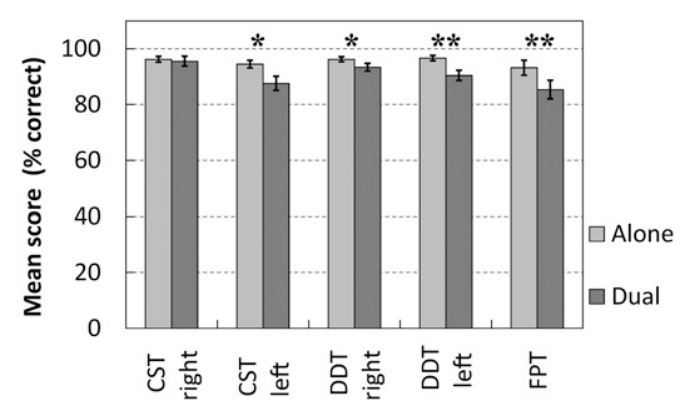

Figure 1. Mean scores of the nonadaptive tests of AP, in alone and dual-task conditions. The figure shows a reduction in score under dual-task conditions for every test (significant in every test except CST right). Error bars indicate standard error of mean $(* p<0.05 ; * * p<0.005)$. presented as SRT in $\mathrm{dB}$ SNR, with more negative scores indicating better performance, that is, participants were able to identify the target signal at poorer signal to noise ratios. Once again, scores in the alone condition are within the normal range for the Australian adult population (Cameron et al, 2011). No significant differences in scores between alone and dual-task conditions were found for any of the adaptive measures of AP, that is, $\mathrm{SV}^{\circ}(Z=1.84, p>0.05), \mathrm{DV}^{\circ}(Z=0.78, p>0.05)$ or $\mathrm{SV}^{\circ} 0^{\circ}(Z=1.24, p>0.05)$.

Figure 3 shows the mean scores of the difference measures "talker advantage" and "spatial advantage" in alone and dual-task conditions. No significant change in score was seen between alone and dualtask conditions for either talker advantage $(Z=0.96$, $p>0.05)$ or spatial advantage $(Z=0.91, p>0.05)$. However, this result is expected, given that the SRTs of the three LiSN-S subtests from which the advantage measures are derived showed no significant differences between alone and dual-task conditions (Figure 2).

\section{Secondary Task Error Analysis}

Errors committed in the secondary (" 3 -odds") task were analyzed as an index of relative task difficulty in the various runs of this task. Figure 4 shows the normalized number of total errors made in the "3-odds" task, when performed alone and in the dual-task condition with each AP test. Total errors include both misses (i.e., no response to presentation of a third consecutive odd digit), and false alarms (i.e., incorrect responses). For every test of AP, except CST Right ear, a significant increase in the errors made in the "3-odds" task was seen, compared with when the "3-odds" task was performed alone; CST Right ear $(Z=1.13, p>0.05)$, CST Left ear $(Z=2.98, p<0.005)$, DDT $(Z=4.46$, $p<0.001)$, FPT $(Z=4.25, p<0.001)$, SV0 ${ }^{\circ}(Z=4.33$, $p<0.001), \mathrm{DV0}^{\circ}(Z=4.18, p<0.001)$ and $\mathrm{SV}^{\circ} 0^{\circ}$ $(Z=4.37, p<0.001)$.

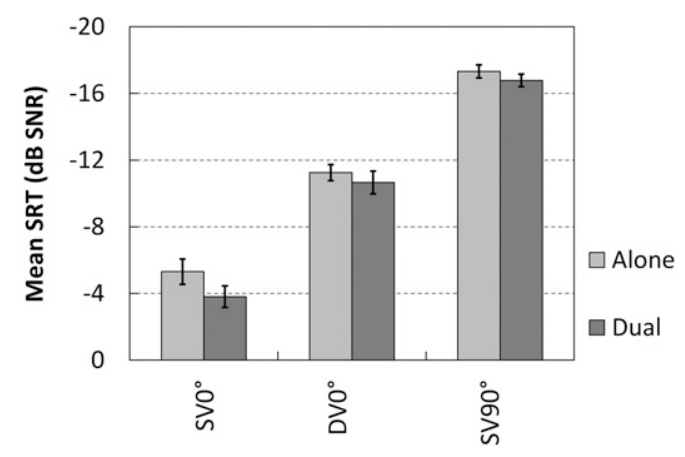

Figure 2. Mean SRT scores on the adaptive tests of AP in alone and dual-task conditions. There are no significant differences between scores in alone and dual-task conditions. Error bars indicate standard error of mean. 


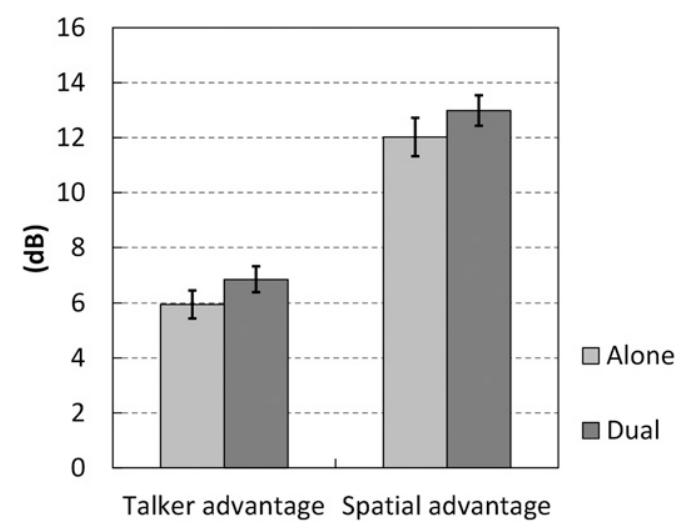

Figure 3. Mean scores of the difference measures of talker and spatial advantages. There are no significant differences between scores in alone and dual-task conditions. Error bars indicate standard error of mean.

The number of errors on the "3-odds" task for CST Right ear was not significantly greater than when completed alone. This is consistent with, and may explain, the lack of change in CST Right ear scores shown in Figure 1. It would suggest that participants had very little difficulty in completing the CST Right ear, with sufficient cognitive spare capacity to simultaneously complete the "3-odds" task at a similar performance level to that when completing the " 3 -odds" on its own. In contrast, Figure 4 shows that errors on the " 3 -odds" task were significantly greater for each of the three LiSN$\mathrm{S}$ subtests than for the "3-odds" task taken alone (and also greater than for most other AP tests). Therefore, unlike the CST Right ear test, the error analysis does not directly explain the lack of change in performance with the increased cognitive demand in the dual-task

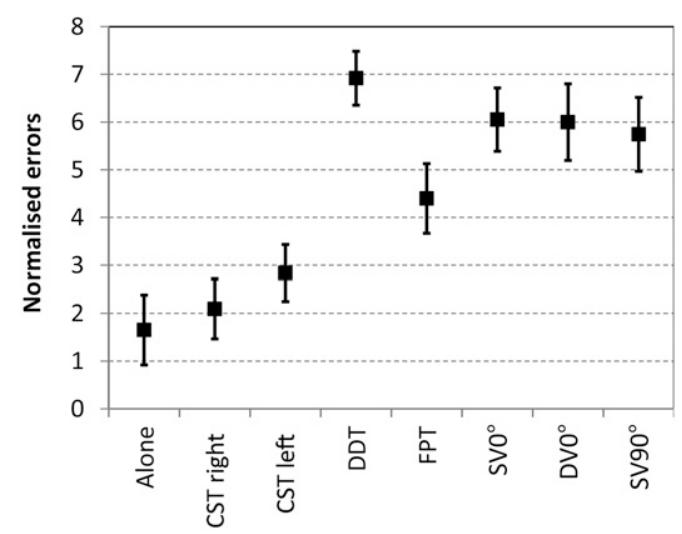

Figure 4. Mean number of (normalized) total errors made in "3odds" task when performed alone and in the dual-task condition with the various AP tests as marked. The number of errors made in the " 3 -odds" task when performed in the dual-task condition is significantly greater than the number of errors on the "3-odds" task when performed alone, for all AP tests except for CST right. Error bars indicate standard error of mean. conditions for the three (adaptive) LiSN-S subtests (Figure 2).

\section{Relationship between Executive Function and Change in AP Test Performance}

Performance on the executive function measure used, VFT total score (mean score $=47$, standard deviation $=$ 13), is in accord with published norms for young Englishspeaking adults with no reported cognitive deficits (Strauss et al, 2006). Some previous studies (e.g., Gates et al, 2010; Foster et al, 2013) have reported an association between measures of executive function and of AP. Therefore, before addressing our final aim (examining the relationship between executive function and change in AP test performance), the relationship between executive function and AP test performance in the alone condition was examined. A significant positive correlation was found between participants' VFT score and both DDT Right (rho $=0.42, p<0.05)$ and DDT Left (rho $=0.39, p<0.05$ ) scores in the alone condition. No significant correlation was found between the executive function measure and the score in the alone condition for any of the other AP tests.

Figure 5 shows the test scores in both alone and dualtask conditions versus the measure of executive function, VFT score, for all four AP measures for which a significant change in performance between alone and dual conditions was obtained. Scores in the alone condition are plotted as filled circles, and those in the dualtask condition as arrowheads; the lengths of the arrows thus represent the changes in AP test score between the two conditions. (The few instances of better scores in the dual-task condition evident [up to $10 \%$ for the CST and DDT, and up to $12 \%$ for the FPT] most likely reflect the test-retest variability. Test-retest repeatability of within 10\% has been reported for the DDT (Strouse and Hall, 1995), but does not appear to have been reported for the CST or the FPT.) There was no significant correlation between the VFT score and the change in AP test score between alone and dual-task condition for any of the AP measures; CST left ear ( $r$ ho $=-0.17, p>$ 0.05), DDT Right ear (rho $=0.31, p>0.05$ ), DDT left ear $($ rho $=-0.15, p>0.05)$, and FPT $($ rho $=0.04$, $p>0.05)$.

\section{DISCUSSION}

11 of the nonadaptive tests of AP, except for CST A Right ear, showed a significant decline in performance in the dual-task condition compared with the alone condition, demonstrating that increased cognitive demand can significantly impact test performance on standard nonadaptive measures of AP. The lack of performance decrement seen for CST Right ear in the dualtask condition likely reflects a strong ceiling effect for 


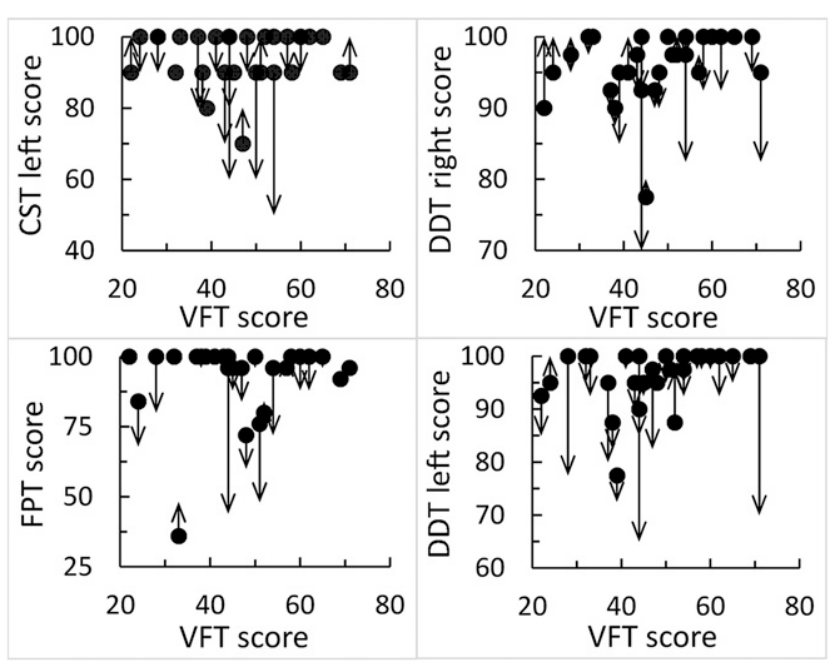

Figure 5. AP test scores in alone (circles) and dual-task (arrowheads) conditions vs. VFT score, for the four AP measures for which a significant change in score between alone and dual conditions was obtained. The length of each arrow represents the change in AP test score between the two conditions. There was no significant correlation between the VFT score and the change in AP test score between alone and dual-task condition for any of the AP measures.

this test, that is, that near-perfect performance in the alone condition was easily achieved by the participants, with sufficient spare capacity, such that near-perfect performance could still be achieved in the dual-task condition. This conclusion is supported both by the scores for the CST Right ear shown in Figure 1, and the minimal change in errors on the " 3 -odds" task when performed simultaneously with the CST Right versus alone, shown in Figure 4. (These data therefore also demonstrate a striking right-ear advantage in young adults with no reported listening difficulties, when cognitive demand is increased by use of the dual-task paradigm. As evident in Figure 1, right ear scores were significantly better than left-ear scores in the dual condition, for both the CST $[Z=2.65 ; p<0.01]$ and the DDT $[Z=2.13 ; p<0.05]$. By contrast, right and left ear scores did not significantly differ (i.e., no rightear advantage was observed) in the alone condition, for either the CST $[Z=1.11 ; p>0.05]$ or the DDT $[Z=0.98 ; p>0.05]$.)

By contrast, none of the scores of the adaptive measures of AP were shown to be significantly affected in the dual-task condition. These results suggest that performance on adaptive tests of AP is less susceptible to cognitive demand (at least as manipulated in this study) than is performance on nonadaptive tests of AP. However, currently the vast majority of commercially available AP tests are nonadaptive in nature, indeed the LiSN-S test appears to be the only widely used commercially available adaptive test of (some aspects of) AP. The present findings therefore support the de- velopment of a new generation of computerized adaptive tests, based on psychophysical principles, that assess the full range of AP abilities (Jerger and Musiek, 2000; ASHA, 2005a; Cameron and Dillon, 2007; O'Beirne et al, 2012). The greater resilience of such tests to cognitive demand, as indicated by these results, would suggest greater validity as measures of the various AP abilities that they are designed to assess.

The difference measures of talker advantage and spatial advantage also showed no significant decrement in score in the dual-task condition. It has been proposed that tests employing control conditions are more resilient to external factors such as language, attention and motivation as these factors are present in both test situations and thus are largely cancelled out when calculating the difference score (Cameron et al, 2011). However, as no statistically significant difference in raw score was seen for any of the LiSN-S subtests $\left(\mathrm{SV}^{\circ}, \mathrm{DV}^{\circ}\right.$, and $\left.\mathrm{SV}^{\circ} 0^{\circ}\right)$ between the alone and dualtask conditions, and talker advantage and spatial advantage are calculated as differences between these raw scores, it is unremarkable that no statistically significant difference in score was seen for either of these difference measures. Therefore, although it seems reasonable that tests employing control conditions would be more resilient to higher-order factors such as cognitive demand, results from this study are unable to provide support for that proposition.

The errors recorded on the "3-odds" task provide an index of difficulty in completing the composite (dual) task. The error analysis shown in Figure 4 does not readily explain the lack of a significant decrement in scores in the dual-task condition for the three adaptive subtests of the LiSN-S. This contrasts with the results discussed previously for the CST Right ear scores. However, the combination of a relatively high proportion of errors on the "3-odds" task with a lack of decrement in scores on the LiSN-S subtests may suggest that participants in fact prioritized these particular AP tests over the "3-odds" task, despite being instructed and reminded to complete both tasks to the best of their ability. (In a similar vein, Choi et al (2008) reported that participants [children] in their dual-task study seemed unable to switch the allocation of priority from a word recognition to a digit recall task.) It is noteworthy that the participants' task in the LiSN-S subtests was broadly similar to that in the CST, that is, to recognize and repeat a target sentence in the presence of competing sentence material. The primary difference between the two (of relevance here) may have been the level of difficulty, which also relates to one being an adaptive and the other a nonadaptive test.

One possibly significant difference between the nonadaptive and adaptive tests of AP in the present study was the brief "down time" (silent period) between a participant's response and the onset of the next trial in the 
nonadaptive tests. As these tests (i.e., CST, DDT, and FPT) were administered from their standard audio recordings, the trial rate was fixed, allowing sufficient time for the participant's response after each trial. Most participants in our study responded well within the interval between trials, leading to the down time referred to above. By contrast, successive trials in the adaptive LiSN-S subtests were generally initiated as soon as a participant's response to the previous trial was scored, leading to less down time in practice. The increased down time associated with the nonadaptive tests may have represented greater potential for participants to switch attention between the two tasks. Attention switching may be central to performance on dual tasks (Pashler, 1994), and may also be more generally important to auditory performance in complex environments (e.g., Dhamani et al, 2013; Carlile, 2014). However, the above differences in down time would be expected to result in a smaller performance decrement in the dual condition in the nonadaptive tests than in the adaptive tests, contrary to the results obtained. The difference in down time between the two types of tests does not therefore explain the greater sensitivity to cognitive demand found for the nonadaptive tests as compared to the adaptive tests. It may, however, have contributed to an increased number of errors on the "3-odds" task in the dual conditions of the adaptive tests. Future studies of this nature should seek to carefully control or eliminate such down time during testing.

Although it may have been expected that individuals with higher executive function may have better dealt with the increased cognitive demands placed on them in the dual-task condition in the present study, the results do not support this expectation. There were no significant correlations between the changes in AP test scores between alone and dual-task conditions, and the measure of executive function used, VFT score. This lack of an association between executive function and AP performance decrements in the dual-task condition may reflect the choice of executive function measure used, and it is possible that another measure, or a combination of measures, may better reflect dual-task performance decrements.

The present findings cannot be directly compared with those of past studies, as there do not appear to be any previous reports of the direct impact of a competing task on AP test performance. However, some general comparisons with the literature on cognitive factors and AP (Table 1) may be appropriate. Firstly, the significant impact of the competing task on performance on the DDT and FPT is consistent with the many previous findings of a link between these tests and cognitive factors such as attention and memory (Sharma et al, 2009; Wilson et al, 2011; Gyldenkærne et al, 2014; Tomlin et al, 2015). However, the present study did not use any gap detection tests, which in previous literature is less frequently linked with cognitive factors. With respect to test properties, whereas the present findings suggest greater resilience of adaptive than nonadaptive tests to cognitive demand, this distinction is not evident in the past literature on the relationship between AP test performance and cognitive measures. Past literature (e.g., Sharma et al, 2009; Gyldenkærne et al, 2014; Tomlin et al, 2015) does suggest that tests that employ control conditions are generally less related to cognitive factors than tests that do not; however, as discussed previously, the present data are unable to shed any light on this comparison. It is emphasized that comparisons of these findings with the past literature are limited by the substantial differences in the approach taken, viz use of a competing task to increase cognitive demand, versus separate measures of cognitive factors. There are also no previous reports of the relationship between the impact of a competing task on AP performance and executive function, with which the present results may be compared. However, the present study's finding that (alone condition) performance on the DDT was significantly associated with the measure of executive function used is consistent with the findings of Gates et al (2010). Those authors found that a composite measure of executive function (derived from a more comprehensive set of measures) was significantly associated with each of three AP measures, including the DDT. The fact that the DDT was the only AP measure in the present study found to demonstrate an association with the executive function measure may suggest that divided attention tasks are particularly dependent on the processes underlying executive function. It is noteworthy that Gates et al (2010) also reported a stronger association between their measure of executive function and their two divided attention AP tasks than their third (monaural) AP task.

Finally, it is acknowledged that these effects of increased cognitive demand on AP assessments as assessed using the dual-task paradigm in young, healthy adults may not directly extend to clinical populations or to other age groups. Further studies are required to draw more direct conclusions concerning the relative susceptibility to cognitive demand of different $\mathrm{AP}$ assessments in, for example, children with reported listening difficulties.

\section{CONCLUSION}

PD is commonly defined as a difficulty in the perA ceptual processing of auditory information in the central nervous system that is not due to higher-order factors. Thus, to accurately identify individuals with the disorder, the tests used in its diagnosis need to be resilient to these higher-order effects. In this study, performance on commonly used nonadaptive tests of AP was shown to be significantly compromised by increased 
cognitive demand, in a dual-task paradigm. These results are consistent with the findings of Moore et al (2010), indicating that poor AP test performance may in practice be attributable to attentional factors. However, performance on AP measures obtained by an adaptive test procedure was not significantly affected by the increased cognitive demand on participants in the dual-task condition. Further investigation of the resilience to increased cognitive demand of the adaptive tests used here, and other adaptive tests of AP, is warranted. Results from this study support the further development of computerized adaptive tests of $A P$, and the use of adaptive tests in clinical AP test batteries.

Acknowledgments. We acknowledge and thank Martin Hamlyn for technical assistance with data analysis.

\section{REFERENCES}

Ahmmed AU, Ahmmed AA, Bath JR, Ferguson MA, Plack CJ, Moore DR. (2014) Assessment of children with suspected auditory processing disorder: a factor analysis study. Ear Hear 35(3):295-305.

American Academy of Audiology (AAA). (2010) Diagnosis, treatment and management of children and adults with central auditory processing disorder. Clinical Practice Guidelines. www.psha. org/pdfs/toolbox/CAPD-Guidelines_8-2010.pdf. Accessed August 26,2016

American Speech-Language-Hearing Association (ASHA). (2005a) (Central) auditory processing disorders [Technical Report]. www. asha.org/policy/tr2005-00043.htm. Accessed August 26, 2016.

American Speech-Language-Hearing Association (ASHA). (2005b) (Central) auditory processing disorders - the role of the audiologist [Position Statement]. www.asha.org/policy/ps2005-00114. htm. Accessed August 26, 2016.

Bellis TH. (2003) Assessment and Management of Central Auditory Processing Disorders in the Educational Setting: From Science to Practice. 2nd ed. New York, NY: Delmar Learning.

Boot WR, Brockmole JR, Simons DJ. (2005) Attention capture is modulated in dual-task situations. Psychon Bull Rev 12(4):662-668.

British Society of Audiology (BSA). (2011) Auditory processing disorder (APD) [Position Statement]. www.thebsa.org.uk/wpcontent/uploads/2014/04/BSA_APD_PositionPaper_31March11_FINAL. pdf. Accessed August 26, 2016.

Cacace AT, McFarland DJ. (2013) Factors influencing tests of auditory processing: a perspective on current issues and relevant concerns. J Am Acad Audiol 24(7):572-589.

Cameron S, Dillon H. (2007) Development of the listening in spatialized noise-sentences test (LISN-S). Ear Hear 28(2):196-211.

Cameron S, Glyde H, Dillon H. (2011) Listening in Spatialized Noise-Sentences Test (LiSN-S): normative and retest reliability data for adolescents and adults up to 60 years of age. J Am Acad Audiol 22(10):697-709.

Carlile S. (2014) Active listening: speech intelligibility in noisy environments. Acoust Aust 42:90-96.

Chermak GD, Tucker E, Seikel JA. (2002) Behavioral characteristics of auditory processing disorder and attention-deficit hyper- activity disorder: predominantly inattentive type. $J$ Am Acad Audiol 13(6):332-338.

Cheung AM, Mitsis EM, Halperin JM. (2004) The relationship of behavioral inhibition to executive functions in young adults. $J$ Clin Exp Neuropsychol 26(3):393-404.

Choi S, Lotto A, Lewis D, Hoover B, Stelmachowicz P. (2008) Attentional modulation of word recognition by children in a dualtask paradigm. J Speech Lang Hear Res 51(4):1042-1054.

Dawes P, Bishop D. (2009) Auditory processing disorder in relation to developmental disorders of language, communication and attention: a review and critique. Int J Lang Commun Disord 44(4):440-465.

de Wit E, Visser-Bochane MI, Steenbergen B, van Dijk P, van der Schans CP, Luinge MR. (2016) Characteristics of auditory processing disorders: a systematic review. J Speech Lang Hear Res 59(2): 384-413.

Dhamani I, Leung J, Carlile S, Sharma M. (2013) Switch attention to listen. Sci Rep 3:1297.

Diamond A. (2013) Executive functions. Annu Rev Psychol 64: 135-168.

Forster KI, Forster JC. (2003) DMDX: a windows display program with millisecond accuracy. Behav Res Methods Instrum Comput 35 (1):116-124.

Foster SM, Kisley MA, Davis HP, Diede NT, Campbell AM, Davalos DB. (2013) Cognitive function predicts neural activity associated with pre-attentive temporal processing. Neuropsychologia 51(2):211-219.

Gates GA, Gibbons LE, McCurry SM, Crane PK, Feeney MP, Larson EB. (2010) Executive dysfunction and presbycusis in older persons with and without memory loss and dementia. Cogn Behav Neurol 23(4):218-223.

Gyldenkærne P, Dillon H, Sharma M, Purdy SC. (2014) Attend to this: the relationship between auditory processing disorders and attention deficits. J Am Acad Audiol 25(7):676-687, quiz 706-707.

Heinrich A, Schneider BA, Craik FIM. (2008) Investigating the influence of continuous babble on auditory short-term memory performance. Q J Exp Psychol (Hove) 61(5):735-751.

Henry JD, Crawford JR. (2004) A meta-analytic review of verbal fluency performance following focal cortical lesions. Neuropsychology 18(2):284-295.

Jerger J, Musiek F. (2000) Report of the consensus conference of the diagnosis of auditory processing disorder in school-aged children. J Am Acad Audiol 11(9):467-474.

Jerger J, Thibodeau L, Martin J, Mehta J, Tillman G, Greenwald R, Britt L, Scott J, Overson G. (2002) Behavioral and electrophysiologic evidence of auditory processing disorder: a twin study. J Am Acad Audiol 13(8):438-460.

Leek MR. (2001) Adaptive procedures in psychophysical research. Percept Psychophys 63(8):1279-1292.

McArthur GM, Bishop DVM. (2004) Frequency discrimination deficits in people with specific language impairment: reliability, validity, and linguistic correlates. J Speech Lang Hear Res 47(3): $527-541$

McCulloch K. (2007) Attention and dual-task conditions: physical therapy implications for individuals with acquired brain injury. $J$ Neurol Phys Ther 31(3):104-118. 
McGarrigle R, Munro KJ, Dawes P, Stewart AJ, Moore DR, Barry JG, Amitay S. (2014) Listening effort and fatigue: what exactly are we measuring? A British Society of Audiology Cognition in Hearing Special Interest Group 'white paper'. Int J Audiol 53(7):433-440.

Moore DR, Ferguson MA, Edmondson-Jones AM, Ratib S, Riley A. (2010) Nature of auditory processing disorder in children. Pediatrics 126(2):e382-e390.

Moore DR, Rosen S, Bamiou DE, Campbell NG, Sirimanna T. (2013) Evolving concepts of developmental auditory processing disorder (APD): a British Society of Audiology APD special interest group 'white paper'. Int J Audiol 52(1):3-13.

Musiek FE. (1983) Assessment of central auditory dysfunction: the dichotic digit test revisited. Ear Hear 4(2):79-83.

Musiek FE. (1994) Frequency (pitch) and duration pattern tests. $J$ Am Acad Audiol 5(4):265-268.

Musiek FE, Chermak GD. (2007) Handbook of (Central) Auditory Processing Disorder: Auditory Neuroscience and Diagnosis. San Diego, CA: Plural Publishing.

O’Beirne GA, McGaffin AJ, Rickard NA. (2012) Development of an adaptive low-pass filtered speech test for the identification of auditory processing disorders. Int J Pediatr Otorhinolaryngol 76(6):777-782.

Pashler H. (1994) Dual-task interference in simple tasks: data and theory. Psychol Bull 116(2):220-244.

PHONAK. (2009) Listening in Spatialized Noise - Sentences Test. LiSN-S Software - User Manual. www.phonakpro.com/content/ dam/phonak/b2b/C_M_tools/Professional_Tools/Diagnostic/LiSNS-User-Manual.pdf. Accessed August 26, 2016.
Sharma M, Purdy SC, Kelly AS. (2009) Comorbidity of auditory processing, language, and reading disorders. J Speech Lang Hear Res 52(3):706-722.

Strauss E, Sherman EMS, Spreen O. (2006) A Compendium of Neuropsychological Tests: Administration, Norms, and Commentary. 3rd ed. New York, NY: Oxford University Press.

Strouse AL, Hall JW, 3rd. (1995) Test-retest reliability of a dichotic digits test for assessing central auditory function in Alzheimer's disease. Audiology 34(2):85-90.

The Canadian Interorganizational Steering Group for SpeechLanguage Pathology and Audiology (CISG). (2012) Canadian Guidelines on Auditory Processing Disorder in Children and Adults: Assessment and Intervention. canadianaudiology.ca/ assets/docs/Canadian_Guidelines_on_Auditory_Processing_Disorder_ in_Children_and_Adults_EN_2012_new-site.pdf. Accessed August 26, 2016.

Tomlin D, Dillon H, Sharma M, Rance G. (2015) The impact of auditory processing and cognitive abilities in children. Ear Hear 36(5):527-542.

Troyer AK, Craik FIM. (2000) The effect of divided attention on memory for items and their context. Can J Exp Psychol 54(3): 161-171.

Vermiglio AJ. (2014) On the clinical entity in audiology: (central) auditory processing and speech recognition in noise disorders. J Am Acad Audiol 25(9):904-917.

Wilson WJ, Jackson A, Pender A, Rose C, Wilson J, Heine C, Khan A. (2011) The CHAPS, SIFTER, and TAPS-R as predictors of (C)AP skills and (C)APD. J Speech Lang Hear Res 54(1): 278-291. 